\title{
Activation of the OVOL1-OVOL2 Axis in the Hair Bulb and in Pilomatricoma
}

Takamichi Ito, ${ }^{\star \dagger}$ Gaku Tsuji, ${ }^{*}$ Fumitaka Ohno, ${ }^{*}$ Hiroshi Uchi, ${ }^{*}$ Takeshi Nakahara, ${ }^{*}$ Akiko Hashimoto-Hachiya, ${ }^{*}$ Yuichi Yoshida, ${ }^{\S}$ Osamu Yamamoto, ${ }^{\S}$ Yoshinao 0da, and Masutaka Furue*

From the Departments of Dermatology* and Anatomic Pathology ${ }^{\dagger}$ and the Division of Skin Surface Sensing, ${ }^{\ddagger}$ Graduate School of Medical Sciences, Kyushu University, Fukuoka; and the Division of Dermatology, ${ }^{\S}$ Department of Medicine of Sensory and Motor Organs, Faculty of Medicine, Tottori University, Tottori, Japan

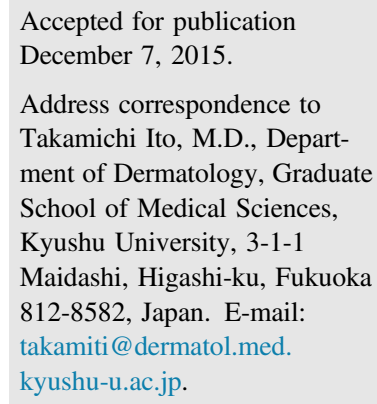

OVOL1 and OVOL2, ubiquitously conserved genes encoding $\mathrm{C} 2 \mathrm{H} 2$ zinc finger transcription factors in mammals, control epithelial cell proliferation, and differentiation, including those in skin. OVOL1 and OVOL2 expression is coordinately mediated via the Wnt signaling pathway, and OVOL1 negatively regulates OVOL2 expression in a transcriptional manner. Our previous study of OVOL1 expression in human skin revealed that OVOL1 is preferentially expressed in the inner root sheath of the hair follicle. Therefore, we hypothesized that the OVOL1-OVOL2 axis is involved in normal and neoplastic follicular differentiation. Immunohistochemical analysis showed that OVOL1 and OVOL2 were strongly expressed in a mutually exclusive manner in the cytoplasm of inner root sheath cells and matrix cells, respectively, in normal follicles. OVOL2 was also expressed in pilomatricoma, with only partial expression of OVOL1. Cultured human keratinocytes expressed OVOL1 and OVOL2 on both the mRNA and protein levels. The expression of OVOL2 was higher in keratinocytes transfected with siRNA of OVOL1. Ketoconazole, a hair growth stimulant, up-regulated the expression of OVOL1 but did not affect OVOL2 expression. These results indicated that the OVOL1-OVOL2 axis may actively contribute to cell differentiation and proliferation in the hair bulb, suggesting that the OVOL1 and OVOL2 may be therapeutic targets of hair disorders, including alopecia, and play important roles in the tumorigenesis of pilomatricoma. (Am J Pathol 2016, 186: 1036-1043; http://dx.doi.org/10.1016/j.ajpath.2015.12.013)
OVOL1 and OVOL2 are ubiquitously conserved genes encoding $\mathrm{C} 2 \mathrm{H} 2$ zinc finger transcription factors in mammals. Functional studies in Caenorhabditis elegans, Drosophila melanogaster, and Mus musculus have suggested that these genes play key roles in the development of epithelial tissues arising from germ cells. ${ }^{1-3}$

OVOL1 is expressed in multiple somatic epithelial tissues, including skin. ${ }^{1}$ Recent studies have shown that the activation of $O V O L 1$ redirects cell proliferation to cell differentiation. ${ }^{3}$ OVOL1 expression is transcriptionally regulated via signal transduction of the lymphoid enhancer factor/ $\beta$-catenin complex in the Wnt signaling pathway, which governs homeostasis, including the development, maintenance, and differentiation of epithelial and hair follicle stem cells. ${ }^{4,5}$

OVOL2 expression has been also observed in murine and human epithelial tissues, such as skin and the germinal epithelium of the testes. ${ }^{6}$ A molecular study of OVOL2 expression demonstrated that $O V O L 1$ represses the activity of the $O V O L 2$ promoter in a DNA-binding-dependent manner. ${ }^{7}$ Furthermore, genetic deletion of OVOL1 up-regulates $O V O L 2$ expression, leading to functional compensation by $O V O L 2$, which suggests that $O V O L 1$ negatively regulates $O V O L 2$ expression.

The OVOL1-OVOL2 axis is reportedly crucial for the normal development and differentiation of hair follicles in a murine model; OVOL1-deficient mice manifest ruffled hair coat and hair abnormalities. ${ }^{1}$ Our previous study showed that human OVOL1 is expressed in the inner root sheath of hair

Supported in part by Ministry of Health, Labor, and Welfare (Japan) grants (M.F.)

T.I. and G.T. contributed equally to this work.

Disclosures: None declared. 

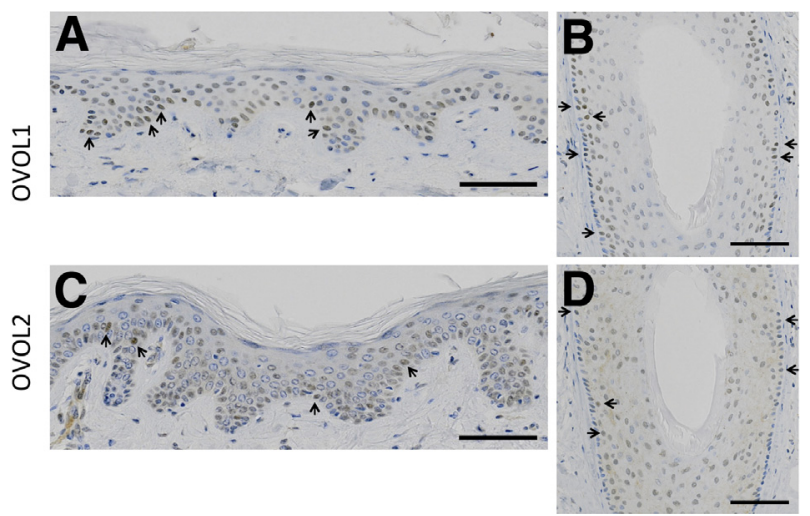

Figure 1 Immunohistochemical staining of OVOL1 (brown staining) and OVOL2 (brown staining) with counter hematoxylin staining in the normal skin and hair follicle. Expression of OVOL1 in basal and suprabasal keratinocytes in the epidermis (A) and the follicular isthmus (arrows; B). The staining patterns are nuclear dominant. Similar staining pattern of OVOL2 in the epidermis (C) and in the follicular isthmus (D). Arrows in A, C, and D indicate keratinocytes expressing OVOL. Scale bars: $100 \mu \mathrm{m}$ (A-D).

follicles, ${ }^{8}$ which is consistent with the confirmed expression of OVOL1 in the hair follicles and suprabasal layer of the epidermis in mice. ${ }^{1}$ Although localization of OVOL2 has not been observed in human hairs, the OVOL1-OVOL2 axis may be involved in differentiation and tumorigenesis, including pilomatricoma in human hair bulbs.

Herein, we found that OVOL1 and OVOL2 are differentially expressed in the normal hair bulb and in pilomatricoma, a common skin tumor derived from the hair matrix. Our results showed that cultured keratinocytes expressed mRNA and proteins of OVOL1 and OVOL2, with up-regulated transcription of OVOL2 under OVOL1 knockdown conditions. Moreover, the hair growth stimulant ketoconazole increased the expression of OVOL1, but not that of OVOL2, implying that ketoconazole might lead to hair growth via OVOL1 expression.

\section{Materials and Methods}

\section{Tissue Samples}

This study was conducted in accordance with the principles of the Declaration of Helsinki and was approved by the Institutional Ethics Committee of Kyushu University Hospital (Fukuoka, Japan). We examined 12 pilomatricoma, 1 pilomatrix carcinoma, and 5 normal skin samples. All formalin-fixed, paraffin-embedded tissues were obtained from the archives of the Department of Dermatology of Kyushu University Hospital and the Division of Dermatology of Tottori University Hospital (Tottori, Japan). Clinical and demographic data were retrieved from patient files. The diagnosis of pilomatrix carcinoma was confirmed through comprehensive assessment with immunohistochemistry and electron microscopy.

\section{Immunohistochemical Analysis}

All samples were fixed with $10 \%$ buffered formalin. The archival paraffin-embedded tissue blocks were cut into sections
( $4 \mu \mathrm{m}$ thick). The sections were deparaffinized with xylene for 10 minutes and rehydrated with a graded ethanol series. Antigen retrieval was performed with Heat Processor Solution pH6 (Nichirei Biosciences Inc., Tokyo, Japan) at $100^{\circ} \mathrm{C}$ for $40 \mathrm{mi}-$ nutes, and endogenous peroxidase was blocked by incubating the sections with $3 \% \mathrm{H}_{2} \mathrm{O}_{2}$ (Nichirei Biosciences Inc.). The sections were then incubated with rabbit antibody against human OVOL1 (1:200; LifeSpan BioSciences, Inc., Seattle, WA), rabbit antibody against human OVOL2 (1:50; Novus Biologicals, Inc., Littleton, CO), mouse antibody against human cytokeratin 5 (CK5; 1:50; Abcam, Inc., Cambridge, UK), or mouse antibody against human trichohyalin (AE15; 1:50; Abcam, Inc.) at $4^{\circ} \mathrm{C}$ overnight, followed by incubation with secondary antibody, N-Histofine Simple Stain MAX-PO MULTI (Nichirei Biosciences Inc.). Immunodetection was conducted with 3,3-diaminobenzidine as a chromogen, followed by light counterstaining with hematoxylin. Sections stained without primary antibody served as the negative control. For immuno-double staining, in addition to the immunodetection using the horseradish peroxidase method mentioned above, N-Histofine Simple Stain AP (Nichirei Biosciences Inc.) and PermaBlue/AP (Diagnostic BioSystems, Inc., Pleasanton, CA) were used as the secondary antibody and the chromogen, respectively.

\section{Cell Culture of NHEKs}

Normal human epidermal keratinocytes (NHEKs) obtained from Lonza Ltd (Basel, Switzerland) were grown in culture dishes at $37^{\circ} \mathrm{C}$ in $5 \% \mathrm{CO}_{2}$. NHEKs were then cultured in serum-free keratinocyte growth medium (Lonza, Walkersville,

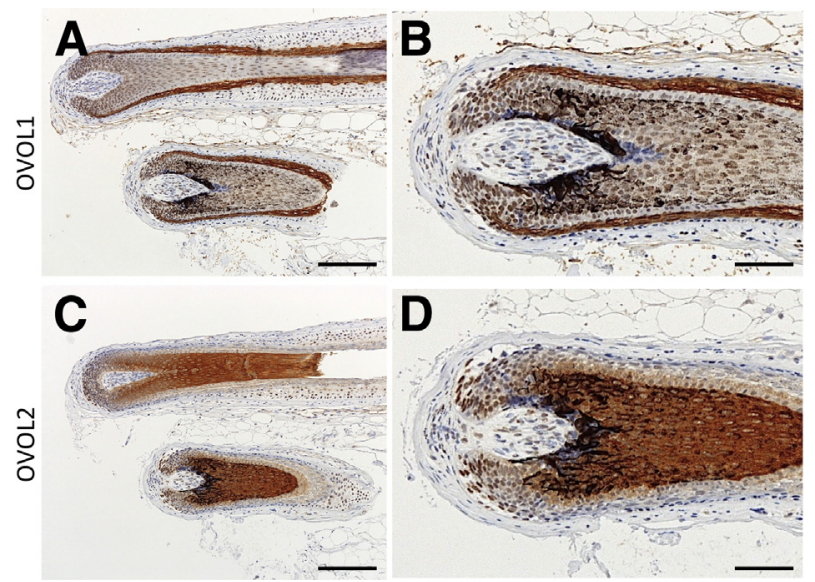

Figure 2 Immunohistochemical staining of OVOL1 (brown staining) and OVOL2 (brown staining) with counter hematoxylin staining in the normal hair bulb. A and B: OVOL1 expression in the normal hair bulb. Positive staining for OVOL1 is observed in cells of the inner root sheath (cytoplasmic), hair germ (nuclear), matrix (cytoplasmic and nuclear), cortex (nuclear), and outer root sheath (nuclear). C and D: OVOL2 expression in the normal hair bulb. Positive staining for OVOL2 is observed in the cytoplasm of cells in the matrix, medulla, and cortex and in the nuclei of hair germ cells and outer root sheath cells. Inner root sheath cells show both cytoplasmic and nuclear staining. Scale bars: $200 \mu \mathrm{m}$ (A and C); $100 \mu \mathrm{m}$ (B and D). 
Table 1 Expression Patterns of OVOL1 and OVOL2 in the Hair Bulb

\begin{tabular}{lll}
\hline Part of hair bulb & OVOL1 & OVOL2 \\
\hline Hair germ cells & Nuclear $(+++)$ & Nuclear $(+++)$ \\
$\begin{array}{l}\text { Medulla } \\
\text { Matrix }\end{array}$ & $(-)$ & Cytoplasmic $(+++)$ \\
& Nuclear $(+)$, & Cytoplasmic $(+++)$ \\
cytoplasmic $(+)$ & Cytoplasmic $(+++)$ \\
Cortex & Nuclear $(+)$ & Nuclear $(+)$, \\
Inner root sheath & Cytoplasmic $(+++)$ & cytoplasmic $(+)$ \\
Outer root sheath & Nuclear $(+)$ & Nuclear $(+)$
\end{tabular}

+ , Expression present; +++ , strong expression;,- no expression.

MD) supplemented with bovine pituitary extract, recombinant epidermal growth factor, insulin, hydrocortisone, transferrin, and epinephrine. The culture medium was replaced every 2 days. When the cells were near confluence (70\% to $90 \%$ ), they were disaggregated with $0.25 \mathrm{mg} / \mathrm{mL}$ trypsin/0.01\% EDTA and subcultured. Second- to fourth-passage NHEKs were used in all experiments.

NHEKs $\left(1 \times 10^{5}\right)$ were seeded in 24-well culture plates, allowed to attach for 24 hours, and treated with ketoconazole or dimethyl sulfoxide. Various concentrations of ketoconazole (up to $10 \mu \mathrm{mol} / \mathrm{L}$ ) were prepared with cell culture medium. Control cultures used media containing comparable amounts of dimethyl sulfoxide (up to $0.02 \%$ ). Fresh medium containing ketoconazole (Sigma-Aldrich, St Louis, MO) or dimethyl sulfoxide (Sigma-Aldrich) was added as indicated in the figure legends.

\section{Immunofluorescence and Confocal Laser-Scanning Microscopic Analysis}

NHEKs cultured on slides were washed in phosphatebuffered saline (PBS), fixed with acetone for 10 minutes, and blocked with $10 \%$ bovine serum albumin in PBS for 30 minutes. Samples were incubated with primary rabbit anti-OVOL1 (1:50), anti-OVOL2 (1:50), or control rabbit $\operatorname{IgG}(1: 100)$ in PBS overnight at $4^{\circ} \mathrm{C}$. Slides were washed in PBS before incubation with anti-rabbit (Alexa Fluor 546 or 488; Molecular Probes, Eugene, OR) secondary antibody for 1 hour at room temperature. After nuclear staining with DAPI, the slides were mounted with UltraCruz mounting medium (Santa Cruz Biotechnology, Santa Cruz, CA). All samples were analyzed with a D-Eclipse confocal laser-scanning microscope (Nikon, Tokyo, Japan).

\section{RT-PCR and Quantitative RT-PCR Analysis}

Total RNA was extracted with an RNeasy Mini kit (Qiagen, Courtaboeuf, France). Reverse transcription was performed with a PrimeScript RT-reagent kit (Takara Bio, Otsu, Japan). Quantitative RT-PCR was performed on a Mx3000p real-time system (Stratagene, La Jolla, CA) with SYBR Premix Ex Taq
(Takara Bio). Amplification was started at $95^{\circ} \mathrm{C}$ for $30 \mathrm{sec}-$ onds as the first step, followed by 40 cycles of quantitative RT-PCR at $95^{\circ} \mathrm{C}$ for 5 seconds and $60^{\circ} \mathrm{C}$ for 20 seconds. mRNA expression was measured in triplicate and normalized to $\beta$-actin expression levels. The primers, from Takara Bio and SABiosciences (Frederick, MD), were as follows: OVOL1 (forward, 5'-ACGATGCCCATCCACTACCTG-3'; reverse, $5^{\prime}$-TTTCTGAGGTGCTGGTCATCATTC- $3^{\prime}$ ), OVOL2 (forward, 5'-GGCAAGGGCTTCAACGACA-3'; reverse, 5'-CTTCAGGTGGGACTCCAGAGA-3'), and $\beta$-actin (forward, 5'-ATTGCCGACAGGATGCAGA-3'; reverse, 5'-GAGTACTTGCGCTCAGGAGGA- $3^{\prime}$ ). The CYP1A1 primers were PPH01271E (SABiosciences).

\section{Western Blot Analysis}

NHEKs were incubated with lysis buffer (Complete Lysis-M; Roche Applied Science, Indianapolis, IN). The lysate protein concentration was measured with a BCA Protein Assay kit (Pierce, Rockford, IL). Equal amounts of protein $(20 \mu \mathrm{g})$ were dissolved in NuPage LDS Sample Buffer (Invitrogen, Carlsbad, CA) and 10\% NuPage Sample Reducing Agent (Invitrogen). Lysates were boiled at $70^{\circ} \mathrm{C}$ for 10 minutes and loaded and run on $4 \%$ to $12 \%$ NuPage Bis-Tris Gels (Invitrogen) at $200 \mathrm{~V}$ for 40 minutes. The proteins were transferred to polyvinylidene fluoride membranes (Invitrogen) and blocked in 2\% bovine serum albumin in $0.1 \%$ Tween-20 (Sigma-Aldrich) and Trisbuffered saline. Membranes were probed with anti-OVOL1, anti-OVOL2, or anti- $\beta$-actin antibodies overnight at $4{ }^{\circ} \mathrm{C}$. The secondary antibody was anti-rabbit horseradish peroxidase-conjugated IgG. Protein bands were detected with a Western Breeze kit (Invitrogen). Densitometric analysis of

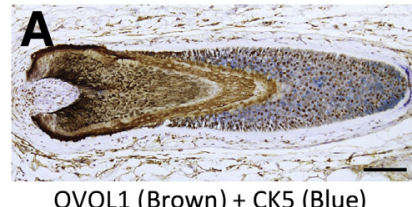

OVOL1 (Brown) + CK5 (Blue)
OVOL2 (Brown) + AE15 (Blue)
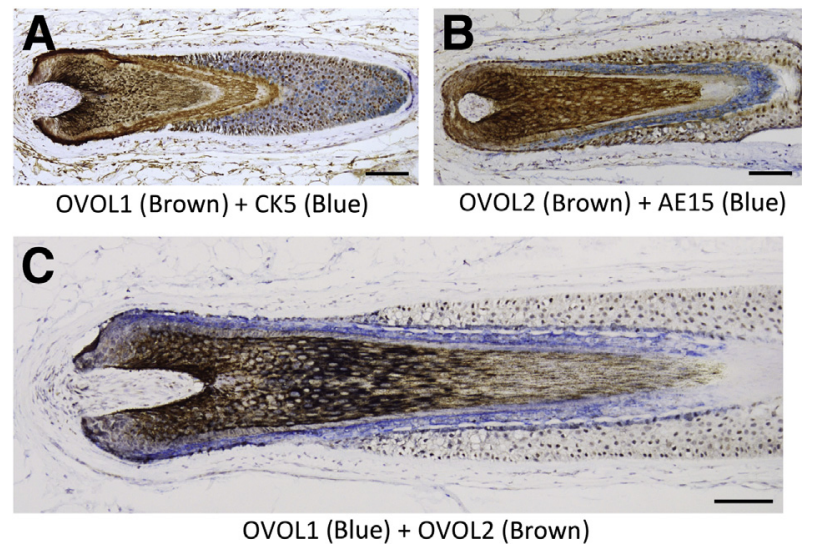

Figure 3 A: Immuno-double staining of OVOL1 (brown staining) and cytokeratin 5 (CK5; blue staining) with counter hematoxylin staining. CK5 stains in cytoplasm of the outer root sheath, which highlights the cytoplasmic staining of OVOL1 in the inner root sheath. B: Immunodouble staining of OVOL2 (brown staining) and trichohyalin (AE15; blue staining). AE15 stains in the cytoplasm of the inner root sheath, which highlights the cytoplasmic staining of OVOL2 in the matrix. C: Double immunostaining of OVOL1 (blue staining) and OVOL2 (brown staining). Cells in the outer root sheath showed nuclear staining of OVOL1, OVOL2, or both. Scale bars: $100 \mu \mathrm{m}(\mathbf{A}-\mathbf{C})$. 

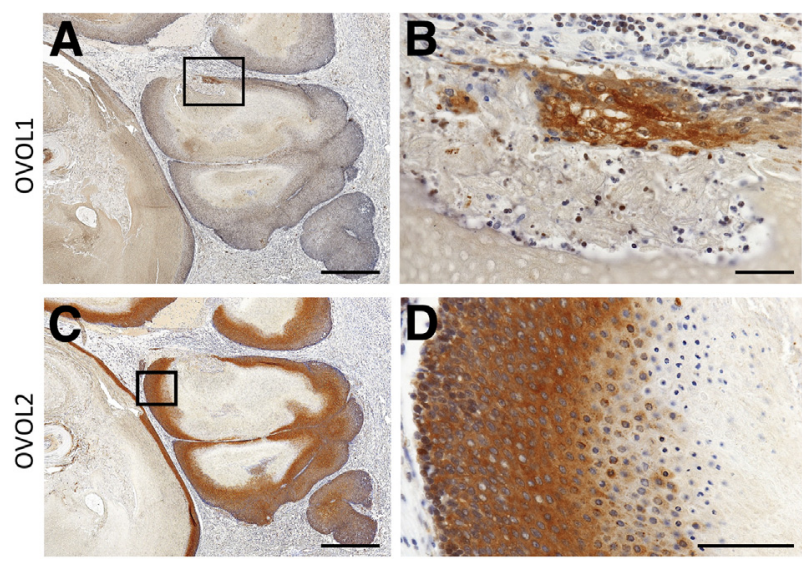

Figure 4 Immunohistochemical staining of OVOL1 (brown staining) and OVOL2 (brown staining) with counter hematoxylin staining in pilomatricoma. Only small OVOL1-positive cell nests are observed. C and D: OVOL2 expression in pilomatricoma. Most of the basaloid cells show strong cytoplasmic expression that gradually fades during the transition into shadow cells. Tumor cells at the periphery of the nests show nuclear staining. The boxed areas in $\mathbf{A}$ and $\mathbf{C}$ are magnified in $\mathbf{B}$ and $\mathbf{D}$, respectively. Scale bars: $500 \mu \mathrm{m}$ (A and $\mathbf{C}) ; 100 \mu \mathrm{m}$ (B and $\mathbf{D})$.

protein bands was performed with ImageJ software version 1.49 (NIH, Bethesda, MD; http://imagej.nih.gov/ij).

\section{Results}

Immunohistological Localization of OVOL1 and OVOL2 in the Hair Bulb and in Pilomatricoma

In agreement with results reported previously, ${ }^{8}$ we detected faint expression of OVOL1 in the basal and suprabasal keratinocytes of normal epidermis with a nuclear-dominant pattern (Figure 1A). Positive signals for OVOL2 were also faintly detected in the basal keratinocytes and in suprabasal cells, mainly with a nuclear pattern (Figure 1C). Similar localization of OVOL1 and OVOL2 was detected in cells of the follicular isthmus (Figure 1, B and D) and sebaceous glands.

In the hair bulb, germ cell nuclei were strongly positive for OVOL1, which maintained moderate expression during transit to the matrix and cortex. Cells in the outer root sheath also stained positively for OVOL1. Abundant cytoplasmic staining was evident in inner root sheath cells (Figure 2, A and B). OVOL2 was also strongly expressed in the nuclei of hair germ cells and cells in the outer root sheath. Notably, strong cytoplasmic staining of OVOL2 (Figure 2C) was observed in cells in the matrix, cortex, and medulla, which was a stark contrast to the strong cytoplasmic staining of OVOL1 in inner root sheath cells (Figure 2A). Weak staining of OVOL2 in the inner root sheath cells was observed in both nuclei and cytoplasm (Figure 2D). The expression patterns of OVOL1 and OVOL2 in the hair bulb are summarized in Table 1.

To further examine whether the OVOL1-OVOL2 axis is involved in the cell differentiation toward outer root sheath and inner root sheath in the hair bulb, we used double immunostaining using OVOL1, OVOL2, CK5, and AE15. Double immunostaining highlighted the localization of OVOL1 and OVOL2 (Figure 3, A and B). AE15 and CK5 strongly stain in the cytoplasm of inner root sheath cells and outer root sheath cells, respectively. Strong cytoplasmic expression levels of OVOL1 in inner root sheath and OVOL2 in matrix were observed (Table 1). Co-expression of OVOL1 and OVOL2 was also observed in the nuclei of outer root sheath cells and germ cells (Figure 3C).

OVOL1 and OVOL2 expression in the pilomatricoma is shown in Figures 4 and 5. Notably, moderate to strong OVOL2 expression was observed in all (12 of 12) of the pilomatricoma samples (Figure 4, C and D). The OVOL2 was expressed in the nuclei of the tumor cell at the periphery of the nests and in the cytoplasm of most of the basaloid cells. Cells in the transitional zone showed weak to mild OVOL2 expression in the cytoplasm, which gradually faded during the transition into shadow cells (Figure 4, C and D). The nuclear OVOL2-positive cells were only focally positive for CK5, suggesting the differentiation toward outer root sheath in part (Figure 5A). Most of the pilomatricoma cells were negative or only faintly positive (nuclear) for OVOL1; small
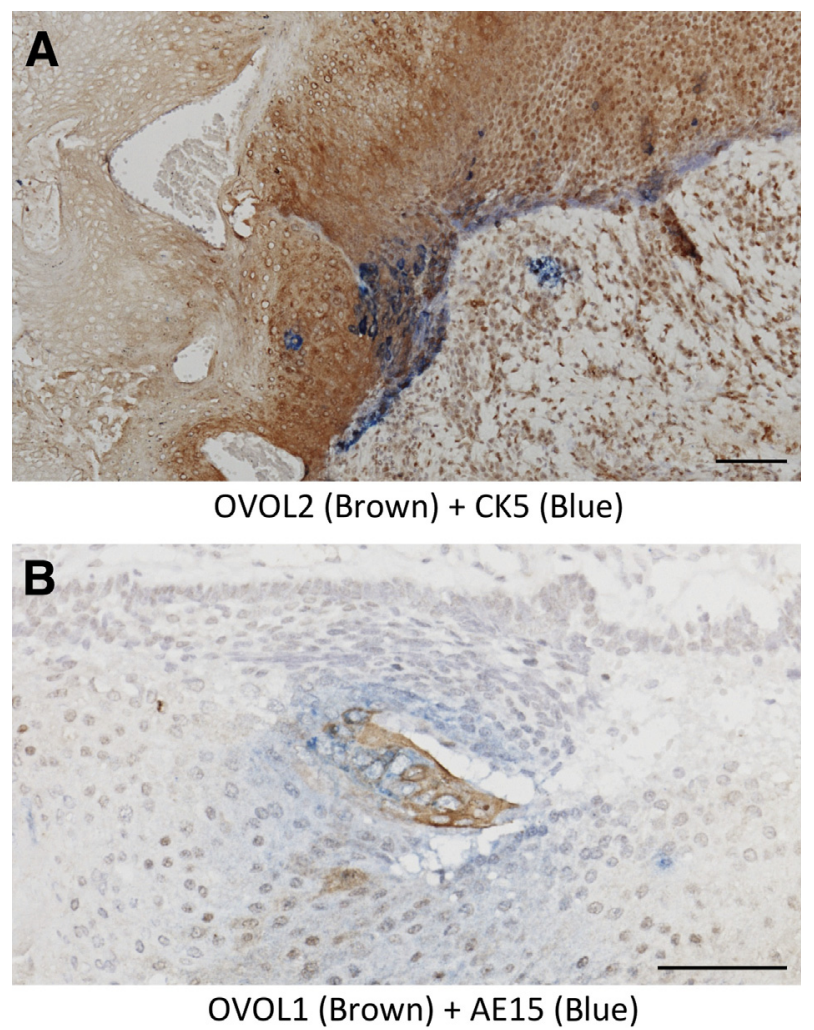

Figure 5 A: Double immunostaining of OVOL2 (brown staining) and cytokeratin 5 (CK5; blue staining) in pilomatricoma with faint counterstaining with hematoxylin. The nuclear OVOL2-positive cells are only focally positive for CK5, suggesting the focal differentiation toward the outer root sheath. B: Double immunostaining of OVOL1 (brown staining) and trichohyalin (AE15; blue staining) in pilomatricoma with faint counterstaining with hematoxylin. OVOL1-positive cells are also positive for AE15, suggesting the differentiation toward the inner root sheath. Scale bars: $200 \mu \mathrm{m}$ (A); $100 \mu \mathrm{m}$ (B). 
Table 2 Expression Pattern of OVOL1 and OVOL2 in Pilomatricoma

\begin{tabular}{|c|c|c|}
\hline Pilomatricoma & OVOL1 & OVOL2 \\
\hline $\begin{array}{c}\text { Periphery of } \\
\text { the nest }\end{array}$ & $(-) /$ Nuclear $(+)^{*}$ & Nuclear $(+++)$ \\
\hline $\begin{array}{l}\text { Transitional } \\
\text { zone }\end{array}$ & $(-) /$ Nuclear $(+)^{*}$ & $\begin{array}{l}\text { Nuclear }(+/+++) \\
\quad \text { cytoplasmic }(+/+++)^{+}\end{array}$ \\
\hline Shadow cells & $(-)$ & $(-)$ \\
\hline
\end{tabular}

*Focally strong cytoplasmic expression.

${ }^{\dagger}$ Expression gradually fades during the transition into shadow cells in a regular manner.

+ , Expression present; +++ , strong expression; -, no expression.

OVOL1-positive (cytoplasmic) cell nests were occasionally detected (Figure 4, A and B). The cytoplasmic OVOL1positive cells were also positive for AE15, suggesting the differentiation toward inner root sheath (Figure 5B). These OVOL1- and OVOL2-positive cells were regularly arranged. The expression patterns of OVOL1 and OVOL2 in pilomatricoma are summarized in Table 2.

To further examine how the OVOL1-OVOL2 axis is regulated in the malignant neoplasm of the hair bulb, we evaluated the distribution of OVOL1 and OVOL2 expression in pilomatrix carcinoma. We found that OVOL1 and OVOL2 were expressed in the nucleus (Supplemental Figure S1). However, further studies are necessary to conclude the expression of OVOL1 and OVOL2 axis in pilomatrix carcinoma, because we could examine a single case of pilomatrix carcinoma, a rare malignant neoplasm.
Expression of OVOL1 and OVOL2 in Cultured Human Keratinocytes

We next examined the expression of OVOL1 and OVOL2 in cultured human keratinocytes. Human keratinocytes expressed the mRNA and proteins of OVOL1 and OVOL2 (Figure 6, A and B). The immunofluorescence study demonstrated nuclear and cytoplasmic distribution of OVOL1 and nuclear distribution of OVOL2 (Figure 6C). A previous murine study showed that the genetic deletion of $O V O L 1$ up-regulates $O V O L 2$ expression. ${ }^{7}$ Because the expression of OVOL1 and OVOL2 was differentially regulated in human hair bulbs, we next examined the mutual regulatory capability of OVOL1 and OVOL2 in human keratinocytes. Compared with those observed after transfection with control siRNA, mRNA levels of OVOL1 and OVOL2 were $28.5 \% \pm 2.4 \%$ and $35.8 \% \pm 2.8 \%$, respectively, lower in keratinocytes transfected with $O V O L I$ siRNA or OVOL2 siRNA (Supplemental Figure S2). Notably, the expression of $O V O L 2$ was up-regulated in the $O V O L 1$ knockdown keratinocytes (Figure 7A). However, the expression of OVOL1 was not significantly affected in $O V O L 2$ knockdown keratinocytes (Figure 7B).

\section{Ketoconazole Up-Regulation of OVOL1 But Not OVOL2 Expression}

To examine further the machinery of OVOL1-OVOL2 axis regulation in keratinocytes, we treated the keratinocytes with various concentrations $(\leq 10 \mu \mathrm{mol} / \mathrm{L})$ of ketoconazole, a potent
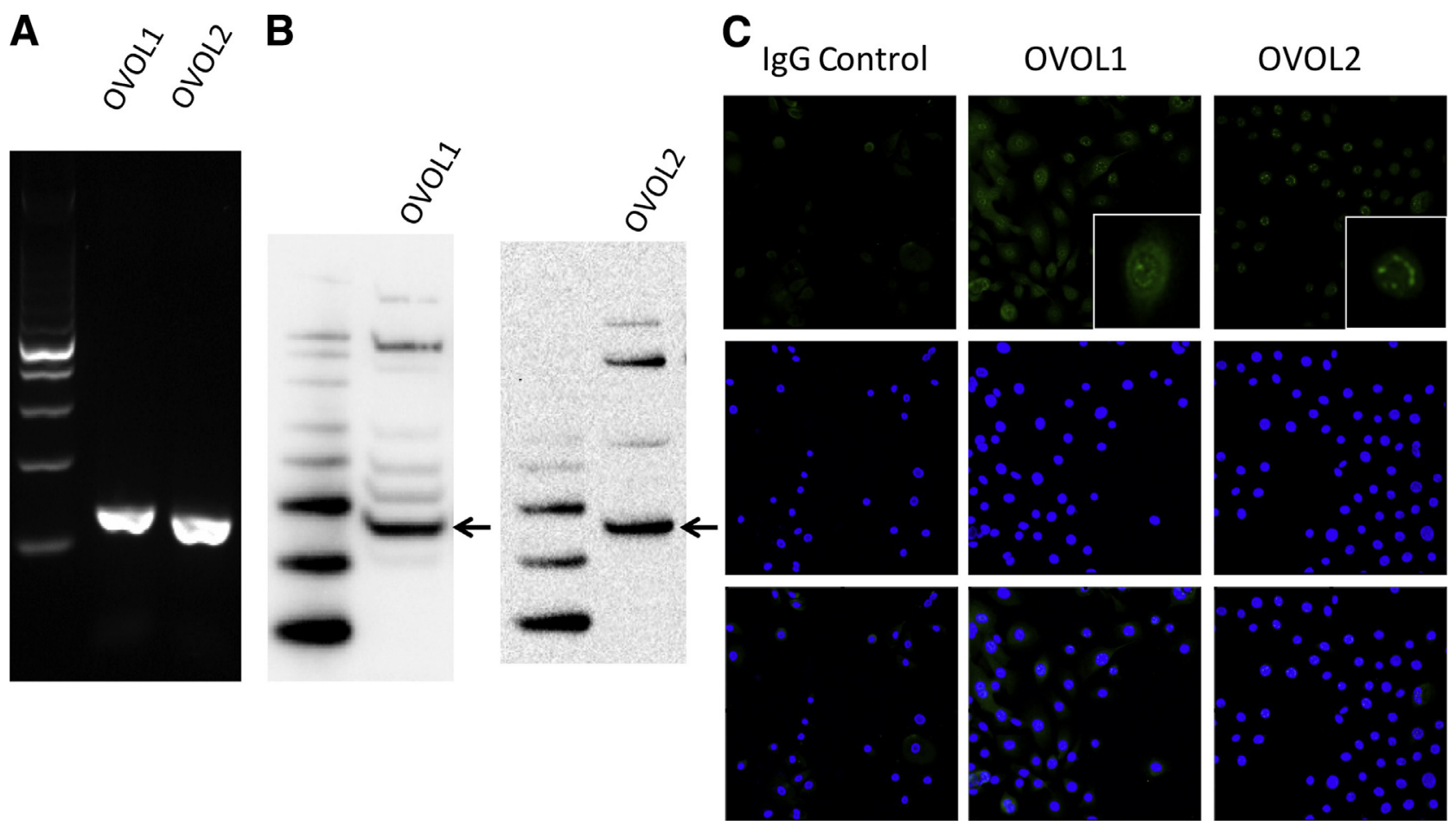

Figure 6 RT-PCR analysis (A) and Western blot analysis (B) of cultured human keratinocytes. Cultured human keratinocytes express the mRNA and proteins (arrows) of OVOL1 and OVOL2. C: The results of the immunofluorescence study demonstrate the nuclear and cytoplasmic distribution of OVOL1 (green) and the nuclear distribution of OVOL2 (green). Blue staining (DAPI) indicates nuclei. Original magnification: $\times 400$ (C, insets). 


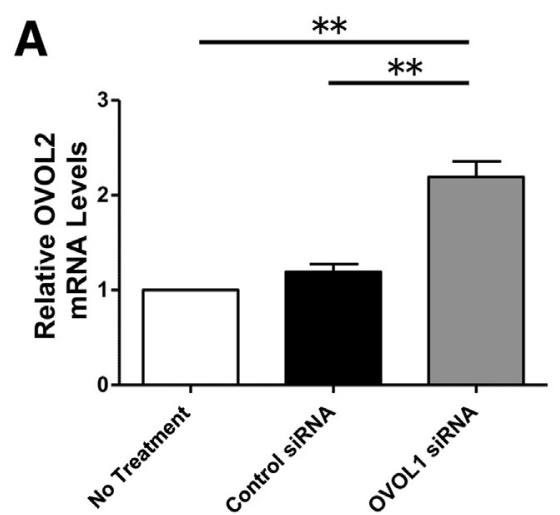

B
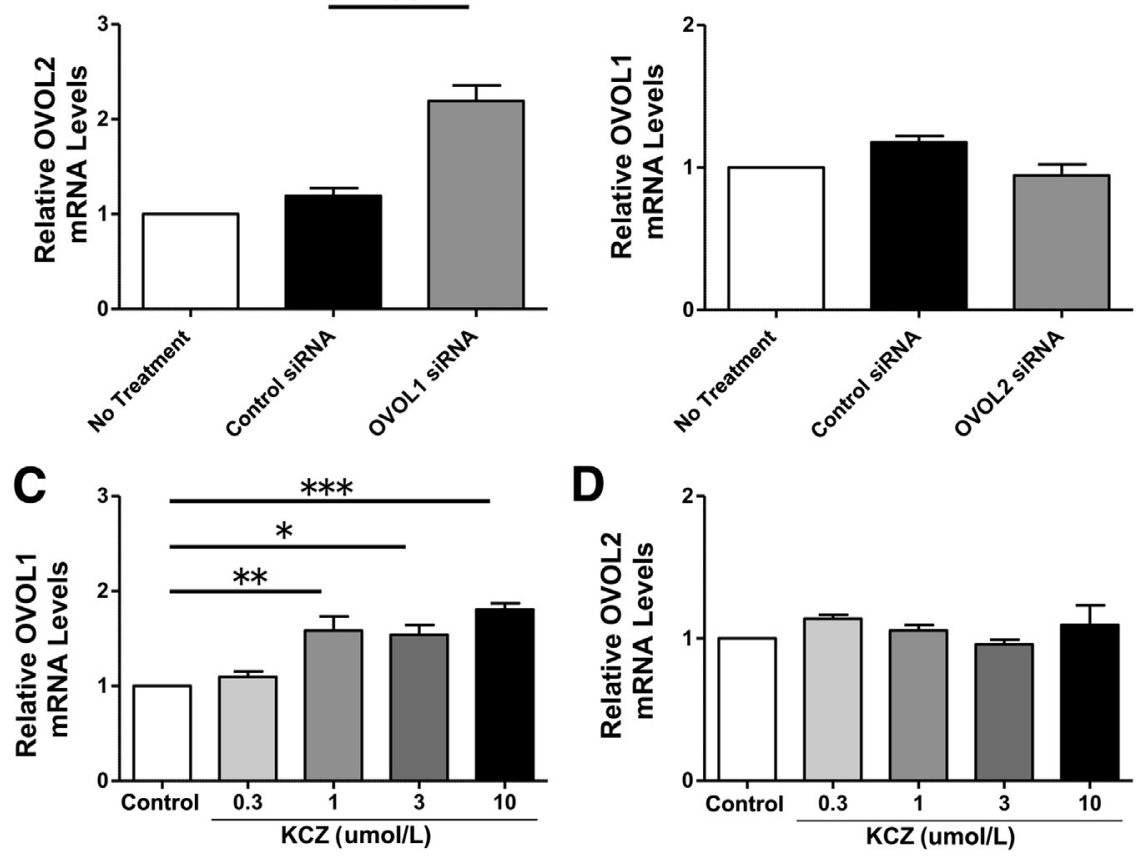

Figure 7 Quantitative RT-PCR analyses of cultured human keratinocytes. A: OVOL2 expression is up-regulated in OVOL1 knockdown keratinocytes. B: OVOL1 expression is slightly decreased in OVOL2 knockdown keratinocytes. C and D: Ketoconazole (KCZ) up-regulates OVOL1 expression in a dose-dependent manner, whereas it has no effect

D

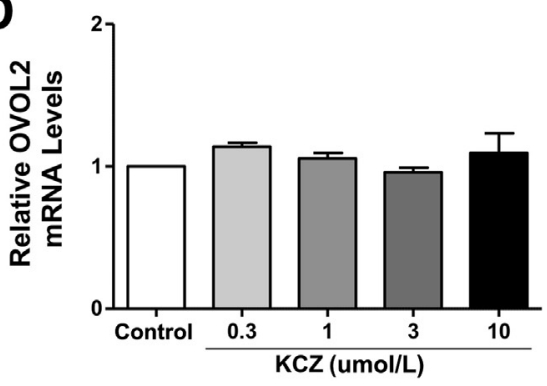
on OVOL2 expression. ${ }^{*} P<0.05,{ }^{*} * P<0.01$, and $* * * P<0.001$.

hair growth stimulant. ${ }^{9,10}$ Ketoconazole up-regulated OVOL1 expression in a dose-dependent manner (Figure 7C), whereas it did not affect the expression of OVOL2 (Figure 7D). The ketoconazole-induced up-regulation of OVOL1 was also evident in protein levels determined with Western blot analysis (Figure 8A). The protein level of OVOL2 was unaffected by ketoconazole. The effects of ketoconazole were also confirmed with immunofluorescence staining. The staining intensity of OVOL1, but not OVOL2, in keratinocytes was again significantly up-regulated by ketoconazole treatment (Figure 8, B and C).

\section{Discussion}

Our results included several key findings about OVOL1 and OVOL2 expression in the hair bulb and in pilomatricoma. OVOL1 and OVOL2 were expressed in normal human skin, especially in the hair bulb. As demonstrated in Figures 2 and 3, the expression of the proteins was highly site specific, with a cytoplasmic pattern, nuclear pattern, or both. Both proteins were expressed in the nuclei of hair germ cells, and expression was maintained during the differentiation toward hair follicles. OVOL1 was specifically observed in the nuclei of cortex and outer root sheath cells, and OVOL2 was present in the nuclei of outer root sheath cells. It is known that OVOL1 is recruited to epidermal differentiation complex genes, leading to promoting keratinocyte differentiation, ${ }^{11}$ and OVOL2 suppresses terminal differentiation of keratinocytes by directly repressing c-myc and Notch-1. The nuclear staining of OVOL1 and OVOL2 in ORS and germ (in addition to above mentioned facts) suggests that OVOL1 and OVOL2 are important transcriptional factors
A

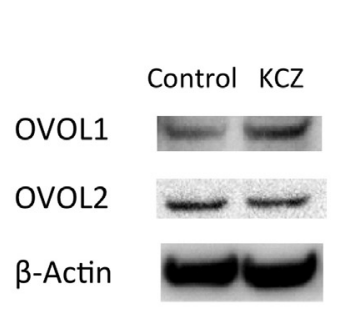

B

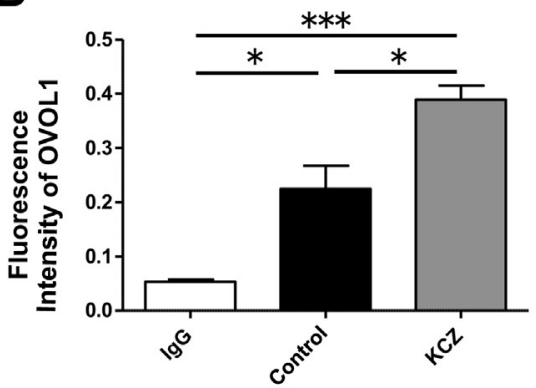

C

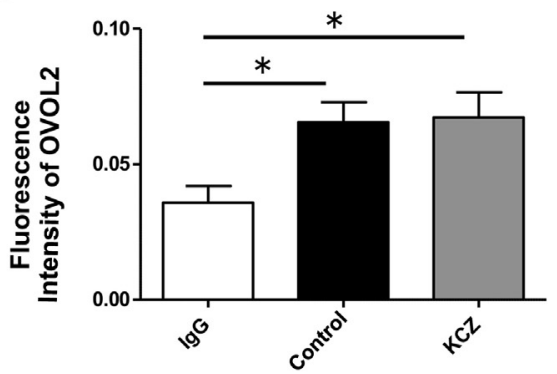

Figure 8 Western blot analysis of cultured human keratinocytes. A: An increased protein level of OVOL1 in keratinocytes treated with ketoconazole (KCZ) is evident compared with that in control. KCZ statistically increases the protein level of OVOL1 (B), but does not affect the OVOL2 protein level (C) in keratinocytes. ${ }^{*} P<0.05,{ }^{* * *} P<0.001$. 
mediating epidermal differentiation complex gene expression and transcriptional activity of c-myc and Notch1 in the hair bulb. ${ }^{12}$ Interestingly, the strong cytoplasmic expression of OVOL1 in the inner root sheath cells contrasted starkly with that of OVOL2 in the cells of the matrix and cortex. The cytoplasmic staining of OVOL1 and OVOL2 was reciprocally exclusive in hair follicle cells (Figures 2 and 3 ). Because OVOL1-deficient mice produce aberrant hair $^{1}$ and OVOL1 inhibits OVOL2 function, ${ }^{7}$ OVOL1 and OVOL2 may coordinately regulate hair differentiation and growth in the development of the hair shaft structure.

Another important observation was that OVOL1 and OVOL2 proteins were expressed in pilomatricoma. Staining for OVOL2 was strongly positive in the cytoplasm of basaloid cells (matrix germinative cells) in pilomatricoma, a hair matrix-derived tumor. This expression faded as cells transitioned into shadow cells. Some basaloid cells at the periphery of the nests showed nuclear staining. The expression pattern closely resembled that of normal hair germ cells and hair matrix cells, which suggests that OVOL proteins may be useful markers for determining the origin of tumors with follicular differentiation. OVOL1, by contrast, showed partial, but strong, cytoplasmic staining in pilomatricoma. These OVOL1-positive cells might differentiate toward inner root sheath cells.

Furthermore, the expression of OVOL1 and OVOL2 with nuclear and cytoplasmic localization is evident in cultured human keratinocytes. Although the cell system using NHEKs might not entirely correspond to keratinocytes of the hair sheath, it has been reported that NHEKs, cultured keratinocytes of the basal layer, and keratinocytes in the lower part of the outer root sheath have similar features that they are positive for keratin 15 and negative for $\mathrm{CD} 34 .{ }^{13}$ A previous murine study demonstrated that Ovol2 is a downstream target of Ovoll by showing that the expression of Ovol2 is up-regulated in Ovoll-deficient epidermis and that Ovoll represses the activity of the Ovol2 promoter in a DNA-binding-dependent manner. ${ }^{7}$ Consistent with this finding, significant up-regulation of OVOL2 was observed in OVOL1-knockdown human keratinocytes in the present study, whereas the expression of OVOL1 was unaffected under the OVOL2 knockdown condition. The clear down-regulation of OVOL2 in OVOL1abundant inner root sheath cells parallels the OVOL1induced repression of $O V O L 2$. In addition, we found that ketoconazole, a potent hair growth stimulator, ${ }^{9,10}$ up-regulated OVOL1, but not OVOL2, expression. Our recent study demonstrated that ketoconazole activates aryl hydrocarbon receptors in keratinocytes, ${ }^{14}$ which implies that OVOL1 acts in concert with the activation of aryl hydrocarbon receptors (AhRs) and leads to hair growth; however, the molecular mechanisms by which AhR activation by ketoconazole upregulates OVOL1 expression remain unclear. Because AhRdependent gene expression requires the nuclear translocation of AhR from cytoplasm to nuclei, ${ }^{15} \mathrm{AhR}$ nuclear transporter (ARNT), a binding partner of the AhR, has a pivotal role in the AhR activation. Considering the facts that the AhR-ARNT system regulates keratinocyte differentiation ${ }^{15}$ and ARNT expression is involved in the hair development, ${ }^{16}$ there is a possibility that OVOL1, in conjunction with the AhR-ARNT system, might play a major role during hair production and development.

These findings, along with those of the present study, suggest that the OVOL1-OVOL2 axis is an active regulator of normal hair development and differentiation. OVOL1 and OVOL2 may be therapeutic targets in the treatment of hair disorders, including alopecia, especially androgenetic alopecia. Furthermore, pilomatricoma conserves the expression of OVOL proteins, and the distribution pattern of this protein expression may be useful in the differentiation of the tumor.

\section{Acknowledgment}

We thank Yuka Eguchi for her assistance with immunohistochemical staining.

\section{Supplemental Data}

Supplemental material for this article can be found at http://dx.doi.org/10.1016/j.ajpath.2015.12.013.

\section{References}

1. Dai X, Schonbaum C, Degenstein L, Bai W, Mahowald A, Fuchs E: The ovo gene required for cuticle formation and oogenesis in flies is involved in hair formation and spermatogenesis in mice. Genes Dev 1998, 12:3452-3463

2. Johnson AD, Fitzsimmons D, Hagman J, Chamberlin HM: EGL38 Pax regulates the ovo-related gene lin-48 during Caenorhabditis elegans organ development. Development 2001, 128: 2857-2865

3. Nair M, Teng A, Bilanchone V, Agrawal A, Li B, Dai X: Ovoll regulates the growth arrest of embryonic epidermal progenitor cells and represses c-myc transcription. J Cell Biol 2006, 173:253-264

4. Li B, Mackay DR, Dai Q, Li TW, Nair M, Fallahi M, Schonbaum CP, Fantes J, Mahowald AP, Waterman ML, Fuchs E, Dai X: The LEF1/beta-catenin complex activates movo1, a mouse homolog of Drosophila ovo required for epidermal appendage differentiation. Proc Natl Acad Sci U S A 2002, 99:6064-6069

5. Lien WH, Fuchs E: Wnt some lose some: transcriptional governance of stem cells by Wnt/ß-catenin signaling. Genes Dev 2014, 28 : $1517-1532$

6. Li B, Dai Q, Li L, Nair M, Mackay DR, Dai X: Ovol2, a mammalian homolog of Drosophila ovo: gene structure, chromosomal mapping, and aberrant expression in blind-sterile mice. Genomics 2002, 80: 319-325

7. Teng A, Nair M, Wells J, Segre JA, Dai X: Strain-dependent perinatal lethality of Ovol1-deficient mice and identification of Ovol2 as a downstream target of Ovol1 in skin epidermis. Biochim Biophys Acta 2007, 1772:89-95

8. Mitoma C, Nakahara T, Uchi H, Ito T, Inatomi $\mathrm{Y}$, Ide $\mathrm{T}$, Jinnai S Jinnai N, Iwasaki N, Sakamoto K, Kimura N, Maeda A, Kuma Y, Maehara E, Tsutsumi M, Kido-Nakahara M, Hirota T, Tamari M, Furue M: Preferential expression of OVOL1 in inner root sheath of hair, sebaceous gland, eccrine duct and their neoplasms in human skin. Fukuoka Igaku Zasshi 2014, 105:166-173 
9. Jiang J, Tsuboi R, Kojima Y, Ogawa H: Topical application of ketoconazole stimulates hair growth in $\mathrm{C} 3 \mathrm{H} / \mathrm{HeN}$ mice. J Dermatol 2005, 32:243-247

10. Aldhalimi MA, Hadi NR, Ghafil FA: Promotive effect of topical ketoconazole, minoxidil, and minoxidil with tretinoin on hair growth in male mice. ISRN Pharmacol 2014, 2014:575423

11. Nascimento EM, Cox CL, MacArthur S, Hussain S, Trotter M, Blanco S, Suraj M, Nichols J, Kübler B, Benitah SA, Hendrich B, Odom DT, Frye M: The opposing transcriptional functions of Sin3a and c-Myc are required to maintain tissue homeostasis. Nat Cell Biol 2011, 13:1395-1405

12. Wells J, Lee B, Cai AQ, Karapetyan A, Lee WJ, Rugg E, Sinha S, Nie Q, Dai X: Ovol2 suppresses cell cycling and terminal differentiation of keratinocytes by directly repressing c-Myc and Notch1. J Biol Chem 2009, 284:29125-29135
13. Kanoh M, Amoh Y, Sato Y, Katsuoka K: Expression of the hair stem cell-specific marker nestin in epidermal and follicular tumors. Eur J Dermatol 2008, 18:518-523

14. Tsuji G, Takahara M, Uchi H, Matsuda T, Chiba T, Takeuchi S, Yasukawa F, Moroi Y, Furue M: Identification of ketoconazole as an AhR-Nrf2 activator in cultured human keratinocytes: the basis of its anti-inflammatory effect. J Invest Dermatol 2012, 132: 59-68

15. Furue M, Takahara M, Nakahara T, Uchi H: Role of AhR/ARNT system in skin homeostasis. Arch Dermatol Res 2014, 306: 769-779

16. Imamura $\mathrm{Y}$, Tomita $\mathrm{S}$, Imanishi $\mathrm{M}$, Kihira $\mathrm{Y}$, Ikeda $\mathrm{Y}$, Ishizawa $\mathrm{K}$, Tsuchiya K, Tamaki T: HIF-2a/ARNT complex regulates hair development via induction of p21(Waf1/Cip1) and p27(Kip1). FASEB J 2014, 28:2517-2524 